\title{
Identification of Binocular Vision Dysfunction (Vertical Heterophoria) in Traumatic Brain Injury Patients and Effects of Individualized Prismatic Spectacle Lenses in the Treatment of Postconcussive Symptoms: A Retrospective Analysis
}

Jennifer E. Doble, MD, Debby L. Feinberg, OD, Mark S. Rosner, MD, Arthur J. Rosner, MD

Objective: To identify a form of binocular vision dysfunction (vertical heterophoria) in a traumatic brain injury (TBI) population and to assess the effect of individualized prismatic spectacle lenses on postconcussive symptoms.

Design: Retrospective study.

Setting: Private physical medicine and rehabilitation practice and private optometric practice.

Patients: A subset of TBI patients who were initially evaluated by a single physiatrist and who received standard treatments and medications yet had persistent postconcussive symptoms. These patients were then assessed by a single optometrist, and those found to have vertical heterophoria were treated with individualized prismatic spectacle lenses. A total of 83 patients were referred for testing; 77 were positive for vertical heterophoria on screening, of which 43 had complete data sets and were included for analysis.

Interventions: All patients were treated with individualized prismatic spectacle lenses to correct for vertical heterophoria.

Main Outcome Measures: Outcomes were measured by the difference in score before and after intervention of an objective, self-administered vertical heterophoria symptom burden instrument (Vertical Heterophoria Symptom Questionnaire [VHS-Q], presently undergoing validation) and by subjective improvement in symptoms as expressed by the patient at the end of intervention.

Results: There was a $71.8 \%$ decrease in subjective symptom burden when compared with preintervention baseline. There was a mean 16.7 point absolute reduction in the VHS-Q score on a 75-point scale, which represents a relative reduction in VHS-Q score of $48.1 \%$.

Conclusion: Vertical heterophoria was identified in a group of TBI patients with postconcussive symptoms and treatment of the vertical heterophoria with individualized prismatic spectacle lenses resulted in a $71.8 \%$ decrease in subjective symptom burden and a relative reduction in VHS-Q score of $48.1 \%$. It appears that vertical heterophoria can be acquired from TBI.

PM R 2010;2:244-253

\section{INTRODUCTION}

It is not uncommon for patients with traumatic brain injury (TBI) to experience cephalgia and neck ache, as well as difficulty with balance, impaired coordination, ambulation problems, anxiety, and vision abnormalities attributable to their TBI [1]. These symptoms can persist even after the patient participates in a prolonged and extensive rehabilitation course. To date, a single unifying cause of these symptoms has not been identified. Vertical heterophoria (VH), a form of binocular vision dysfunction, is associated with a set of symptoms that overlaps significantly with the symptoms associated with TBI (Table 1). At present, an association between VH and TBI is lacking. Serendipitously, a patient experi-
J.E.D. St. Joseph Mercy Hospital, Ann Arbor, Ml; and Special Tree Rehabilitation Systems, Romulus, MI.

Disclosure: nothing to disclose

D.L.F. Vision Specialists of Birmingham, Birmingham, MI

Disclosure: nothing to disclose

M.S.R. Department of Emergency Medicine, University of Michigan, Ann Arbor, Ml; and Department of Emergency Medicine, St. Joseph Mercy Hospital, Ann Arbor, Ml. Address correspondence to: M.S.R.; e-mail: msr50@ comcast.net

Disclosure: nothing to disclose

A.J.R. Department of Otolaryngology, William Beaumont Hospital, Troy, Ml Disclosure: nothing to disclose

Submitted for publication July 10, 2009; accepted January 16, 2010. 
Table 1. Symptoms and differential diagnosis of $\mathrm{VH}$

\begin{tabular}{|c|c|}
\hline Symptom type & Differential diagnosis \\
\hline \multicolumn{2}{|l|}{ Pain } \\
\hline Headache & Migraine headache \\
\hline Face ache/"sinus" pain & Sinusitis \\
\hline \multirow{2}{*}{$\begin{array}{l}\text { Eye pain or pain with eye } \\
\text { movements }\end{array}$} & TMJ \\
\hline & $\begin{array}{l}\text { Chronic daily headache } \\
\text { TBI/postconcussion } \\
\text { syndrome }\end{array}$ \\
\hline \multicolumn{2}{|l|}{ Head tilt } \\
\hline \multirow{3}{*}{$\begin{array}{l}\text { Neck ache and upper back pain } \\
\text { caused by a head tilt }\end{array}$} & CN 4 lesion/SO palsy \\
\hline & Scoliosis \\
\hline & Torticollis \\
\hline \multicolumn{2}{|l|}{ Dizziness } \\
\hline Dizziness & Benign positional vertigo \\
\hline Lightheadedness & Meniere disease \\
\hline Off-balanced & Visual vertigo \\
\hline Motion sickness (is frequently the & Psychogenic dizziness \\
\hline $\begin{array}{l}\text { first symptom of } \mathrm{VH} \text {, can occur } \\
\text { very early in childhood) }\end{array}$ & $\begin{array}{l}\text { Chronic subjective } \\
\text { dizziness }\end{array}$ \\
\hline Vertigo & CVA \\
\hline Nausea & Neuromuscular \\
\hline Poor depth perception & weakness \\
\hline Lack of coordination & Brain tumor \\
\hline $\begin{array}{l}\text { Unsteadiness or drifting to one } \\
\text { side while walking }\end{array}$ & $\begin{array}{l}\text { TBI/postconcussion } \\
\text { syndrome }\end{array}$ \\
\hline $\begin{array}{l}\text { Difficulty walking down grocery } \\
\text { aisle }\end{array}$ & $\begin{array}{l}\text { Migraine-associated } \\
\text { vertigo }\end{array}$ \\
\hline Disorientation & Cervical vertigo \\
\hline & SSCD \\
\hline \multicolumn{2}{|l|}{ Reading } \\
\hline Difficulty with concentration & Reading or learning \\
\hline Fatigue with reading & disability \\
\hline Difficulty with reading and & ADD/ADHD \\
\hline reading comprehension & Convergence \\
\hline Skipping lines while reading & insufficiency \\
\hline $\begin{array}{l}\text { Using a line guide (finger, ruler, } \\
\text { envelope) to maintain one's }\end{array}$ & $\begin{array}{l}\text { Binocular vision } \\
\text { abnormality }\end{array}$ \\
\hline place while reading & Astigmatism \\
\hline Words running together while & Hyperopia \\
\hline reading & TBI/postconcussion \\
\hline Losing one's place while reading & syndrome \\
\hline \multicolumn{2}{|l|}{ Routine visual } \\
\hline Blurred vision at near or far & Myopia \\
\hline distances & Hyperopia \\
\hline $\begin{array}{l}\text { Difficulty with close-up vision (ie, } \\
\text { reading or computer use) }\end{array}$ & Astigmatism \\
\hline Difficulty with night vision & \\
\hline Eye strain & \\
\hline Sore eyes & \\
\hline \multicolumn{2}{|l|}{ Binocular vision } \\
\hline Double or overlapping vision & CVA \\
\hline Shadowed vision & Neuromuscular \\
\hline Light sensitivity & weakness \\
\hline Difficulty with glare or reflection & Brain tumor \\
\hline $\begin{array}{l}\text { Closing/covering one eye while } \\
\text { reading }\end{array}$ & $\begin{array}{l}\text { TBI/postconcussion } \\
\text { syndrome }\end{array}$ \\
\hline
\end{tabular}

Table 1. Continued

\begin{tabular}{cl}
\hline \multicolumn{1}{c}{ Symptom type } & Differential diagnosis \\
\hline Psychological symptoms & \\
Feeling overwhelmed or anxious & Anxiety \\
in crowds & Psychogenic dizziness \\
Agoraphobia & Depression \\
Feeling overwhelmed or anxious & Agoraphobia \\
when in large contained spaces & Chronic subjective \\
like malls or big box stores & dizziness \\
& TBl/postconcussion \\
& syndrome \\
\hline
\end{tabular}

$\mathrm{ADD} / \mathrm{ADHD}=$ attention deficit disorder/attention deficit hyperactivity disorder; CVA = cerebrovascular accident; SSCD = superior semicircular canal dehiscence; $\mathrm{TBI}=$ traumatic brain injury.

enced a marked reduction in presumed postconcussive symptoms after treatment by one of the authors (D.L.F.). Given this positive experience, additional patients were evaluated and treated, with similar results. It is this anecdotal experience that prompted this retrospective analysis and report.

$\mathrm{VH}$ describes a visual condition in which the line of sight from one eye is higher than the line of sight from the other eye when at physiologic rest (an ocular posture created by disrupting fusion with a Maddox Rod or prism; Figure 1). To avoid diplopia when binocular vision is attempted, those with VH excessively use their elevator and depressor extraocular muscle to realign the lines of sight and maintain a fused image. This alignment leads to extraocular muscle strain and fatigue, which is causative of the symptoms (see Table 1 and the Discussion section) [2-7]. Treatment involves individualized spectacle lenses that incorporate vertical prism to correct the $\mathrm{VH}$.

\section{METHODS}

\section{Study Design and Subjects}

This study was approved by the St. Joseph Mercy Health System Institutional Review Board in Ann Arbor, Michigan. A retrospective search of the optometrist's database between January 2005 and April 2008 identified 83 TBI patients that had been referred by one of the authors (J.E.D.). These patients had remained symptomatic with postconcussive symptoms despite receiving standard treatments and medications as directed by this physiatrist and the patient's specialty consultants during a period of months to years. VH was diagnosed by the use of a diagnostic and therapeutic process developed by an optometrist (D.L.F.), whereby prism is added to the patient's baseline prescription in small increments until VH-related symptoms are maximally reduced and maximal comfort is achieved. Records from 83 referred TBI patients were screened for VH. Forty patients were excluded for the following reasons: incomplete records $(\mathrm{n}=$ 20); lost to follow-up or did not complete treatment $(\mathrm{n}=8)$; was not diagnosed by the optometrist with $\mathrm{VH}$ either because history was not consistent with $\mathrm{VH}$ or the patient did not respond to prism challenge ( $\mathrm{n}=5$ ); patient had not com- 


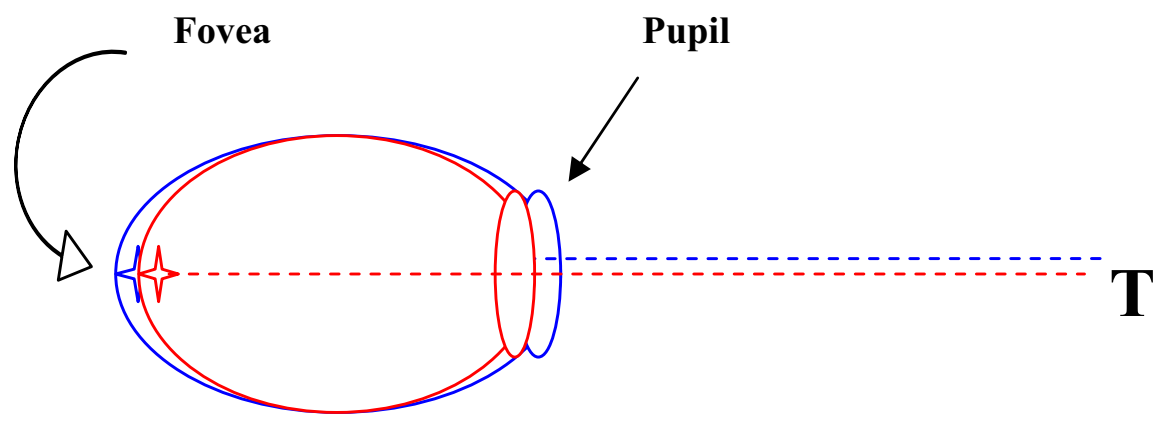

A. Orthophoria

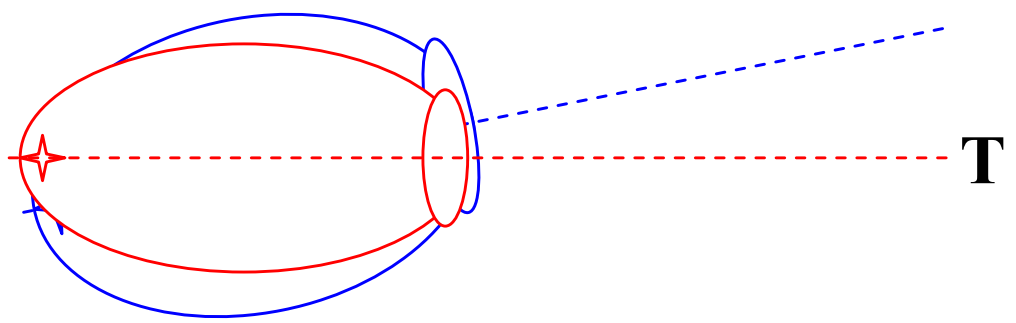

\author{
B. Traditional \\ Vertical Heterophoria \\ due to \\ CN 4 injury / SO palsy
}

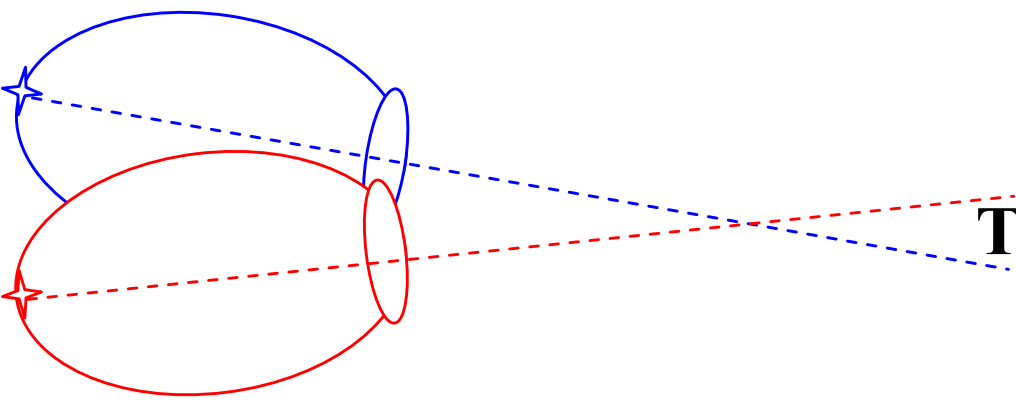

C. Vertical Heterophoria due to

vertical orbital misalignment

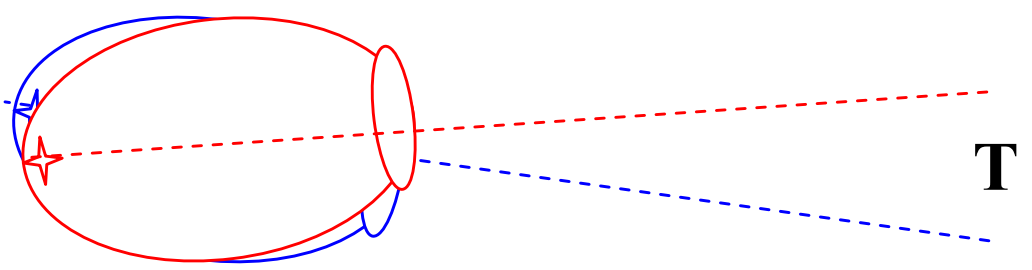

\title{
D. Vertical Heterophoria due to TBI
}

Figure 1. Phoric eye posture (line of sight) in all forms of $\mathrm{VH}$. All types of $\mathrm{VH}$ share the fact that the line of sight from one eye is higher than the line of sight from the other eye. However, there are differences in the phoric eye posture (and therefore the position of the image seen) on the basis of the etiology of the $\mathrm{VH}$. (A) illustrates orthophoria. In traditional $\mathrm{VH}$ (B), the higher eye has an elevated line of sight and sees a lower image, whereas in VH caused by vertical orbital misalignment (C) and in VH due to TBI (D), which are optically equivalent, the line of sight of the higher eye is depressed and the eye sees a higher image. Etiology of "high eye" in (C) is orbital asymmetry, whereas etiology of "high eye" in (B) and (D) is head tilt.

Figure key: Because of the ease of illustration and equivalent nature of the optics, all illustrations of VH will use the Vertical Orbital Misalignment model (Figure 1C). Illustrations are oriented sagittally from the right side of the head. The "high eye" is defined as the eye that is physically higher as the result of orbital asymmetry or the eye that is higher as the result of a head tilt. Images in blue represent the physically higher eye, which is the left eye. Images in red represent the physically lower eye, which is the right eye. Dotted lines represent the phoric position (line of sight) of the eye. Solid lines represent the pathway taken from the target image to the retina through the center of the pupil. $\mathrm{T}=$ target image; FP = focal point. 
Table 2. Demographics

Mean age
44.4 years (12.6)
Gender
M, 28\%/F, 72\%
Cause of TBI
MVC $=40$ (93\%); fall = 1 (2.3\%); blunt injury = 1 (2.3\%);
unknown $=1$ (2.3\%)
Major symptoms*
Headache/face pain/eye pain or strain (33 responses)
Dizziness/vestibular symptoms (21 responses)
Vision symptoms such as blurred vision, diplopia, or reading
difficulties (20 responses)
Neck and shoulder pain (12 responses)
Miscellaneous symptoms (8 responses)
Mean duration of symptoms
3.6 years
Mean duration of treatment
3.6 months

*Patients were asked to list their worst symptoms. Ninety-four responses were obtained from 43 patients.

$\mathrm{MVC}=$ motor vehicle crash; $\mathrm{TBI}=$ traumatic brain injury.

pleted treatment at the time of data collection $(n=7)$. The 43 remaining patients were included in the analysis.

Baseline data were collected on patient age, gender, cause of TBI, major symptoms, duration of symptoms, and duration of treatment (Table 2). Patients were queried at the beginning and at the end of prismatic lens treatment with the use of an objective, self-administered survey instrument developed by one of the authors (D.L.F.) and collaborators (Vertical Heterophoria Symptom Questionnaire [VHS-Q]) to assess the extent of VH symptom burden and to denote changes in symptom burden with intervention. This survey is currently undergoing validation. Patients were also asked to subjectively quantify the percentage improvement in symptoms from before treatment to after completion of treatment with prismatic lenses.

The primary outcome measure was the mean improvement on the VHS-Q symptom questionnaire, and the secondary outcome was the mean percentage of subjective improvement in symptoms relative to baseline. Patient demographic data were summarized by the use of means and percentages. Outcomes were summarized as the mean and SD of improvement in VHS-Q score, and the mean percentage and SD of improvement in symptoms as estimated by the patient. A Spearman rank correlation was performed between relative improvement on the VHS-Q and the estimated percentage of improvement with the use of Spearman rank correlation as a check on validity. We tested whether the VHS-Q difference score was significantly different from 0 by use of the signed rank test. Significance was set at $P<.05$.

\section{RESULTS}

Forty-three patients had complete data sets that were subjected to analysis. Mean baseline VHS-Q score was 34.8

points (SD, 16.1) on a scale of 0 to 75 points. The mean difference in VHS-Q score from before to after prism treatment was 16.7 points (12.8) with a range of -1 to 52 points. This result was significantly different from $0(P<.01)$. The mean subjective improvement in symptoms from baseline was $71.8 \%$ (25\%) (Table 3). Relative improvement on the VHS-Q and the estimated percentage of improvement were correlated $(\mathrm{r}=0.31 ; P=.04)$.

\section{DISCUSSION}

\section{VH Pathophysiology and Symptomatology}

The pathophysiology of $\mathrm{VH}$ is the key to understanding the symptom complex precipitated by this condition, as well as why treatment with prismatic lenses successfully reduces symptoms. Our understanding of the pathophysiology has been derived from the clinical observations of more than $4000 \mathrm{VH}$ patients. It is our hypothesis that in the TBI patient, the brain injury results in the generation of a faulty alignment signal that is vertically misaligning the lines of sight of the eyes (Figure 1D and Figure 2). Although the specific locus or loci of the brain injury responsible for precipitating these symptoms has yet to be identified, the utricle and the brainstem are likely areas.

In response to the misalignment from the aberrant signal, we hypothesize that other visual alignment reflexes activate the opposing elevator and depressor eye muscles to correct the misaligned lines of sight, thereby preventing vertical diplopia and maintaining a fused binocular image (Figure 3). These opposing forces cause the elevator and depressor eye muscles to be under constant undue tension, creating the extraocular muscle strain [8] that is observed clinically. The patients' symptoms of strain are headache and eye pain (asthenopia), which are frequently worsened with eye movement. The extraocular muscle strain eventually leads to extraocular muscle fatigue. These fatigued extraocular muscles are hypothesized to tremor, which would cause the eyes to move minutely in an uncoordinated fashion, making it impossible to consistently maintain fusion (expressed by some patients as "letters moving on the page").

Table 3. Results

Number of patients

Range of possible VHS-Q scores

VHS-Q score before treatment

Mean difference in VHS-Q score from

before to after prism treatment

Relative percent reduction in VHS-Q

score

Mean subjective improvement from before to after prism treatment

Results are mean $( \pm S D)$.

*Range of -1 to 52 points.

${ }^{\dagger}$ Range of $5 \%$ to $100 \%$.

\section{3}

$0-75$ points

34.8 points (16.1)

16.7 points $(12.8)^{*}$

$P<.01$

48.1

$71.8(25)^{\dagger}$ 


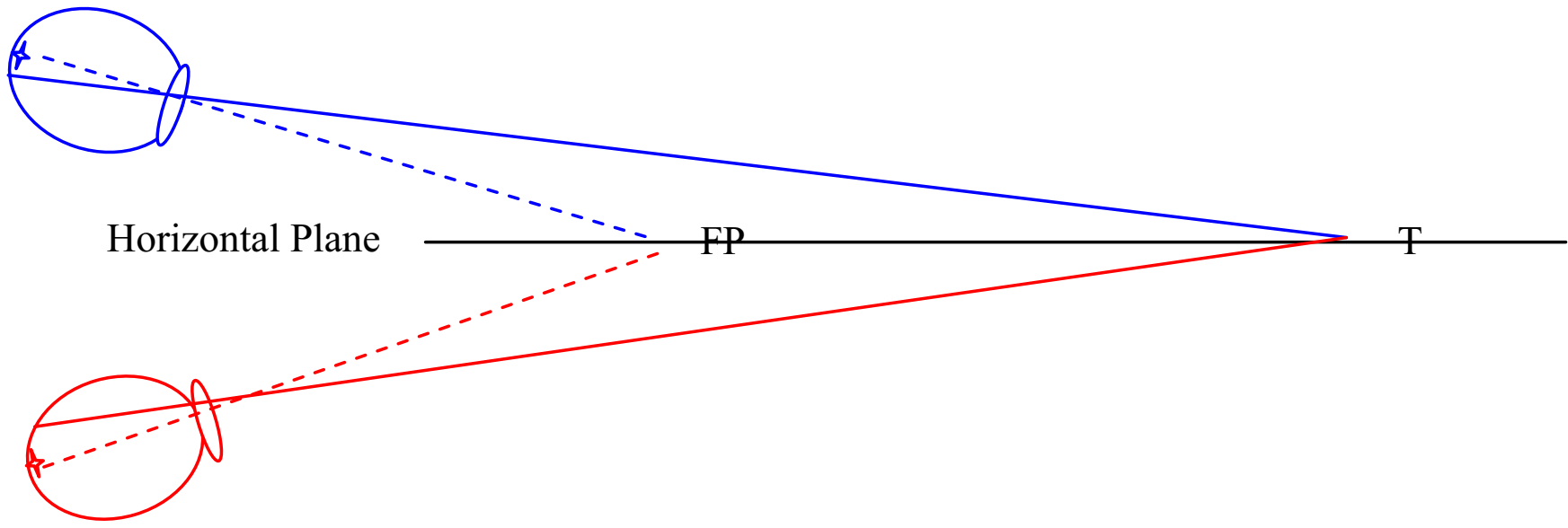

Figure 2. Phoric position of the eyes in vertical transphoria (newly described phoric position). The lines of sight (phoric position) of the eyes are vertically misaligned-they are crossed or transphoric. The "physically higher" eye has the target image striking the retina below the fovea, which is interpreted by the brain as a high image. This will cause blurred vision or vertical diplopia if not corrected. (Elucidated from the position of the head tilt, the position of the eye, the location of the image, and from the direction of correcting vertical prism.) See figure key in Figure 1.

These circumstances would result in the transient diplopia or blurring of the image that is observed clinically [7]. In the stationary individual, the fluctuating, inconsistent visual input would be interpreted by the brain as representing movement. This would be in conflict with the inputs from the vestibular and proprioceptive systems, which would not denote movement. These mismatching inputs would be perceived as dizziness. Appropriate vertical prismatic lenses reposition the lines of sight such that correcting visual alignment reflexes should no longer be needed (Figure 4), which would then allow for the elimination of the undue tension and fatigue in the vertical aligning eye muscles, thus explaining the reduction or elimination of symptoms that is observed clinically.

There are additional symptoms associated with VH. Dizziness is well known to precipitate anxiety [9-11]. Head tilt is a known compensatory mechanism that occurs in $\mathrm{VH}$ to minimize vertical image disparity and avoid diplopia (Figure 5) $[3,7]$. Routine visual symptoms, reading symptoms, and binocular vision symptoms (Table 1) are all well known to be precipitated by heterophoria [7].

\section{History of VH}

A review of the literature demonstrates that VH was first described by Stevens in 1887 [12]. Attempts by Stevens to treat the vertical misalignment with 1.75 diopter (D), 3.5 D, $5.25 \mathrm{D}$, and $7 \mathrm{D}$ vertical prismatic lenses ("large amounts") were largely unsuccessful, but some success was achieved with surgical intervention (extraocular muscle tenotomy). These results, however, appear to have been difficult to reproduce. In the 1950s, Roy was successful in diagnosing

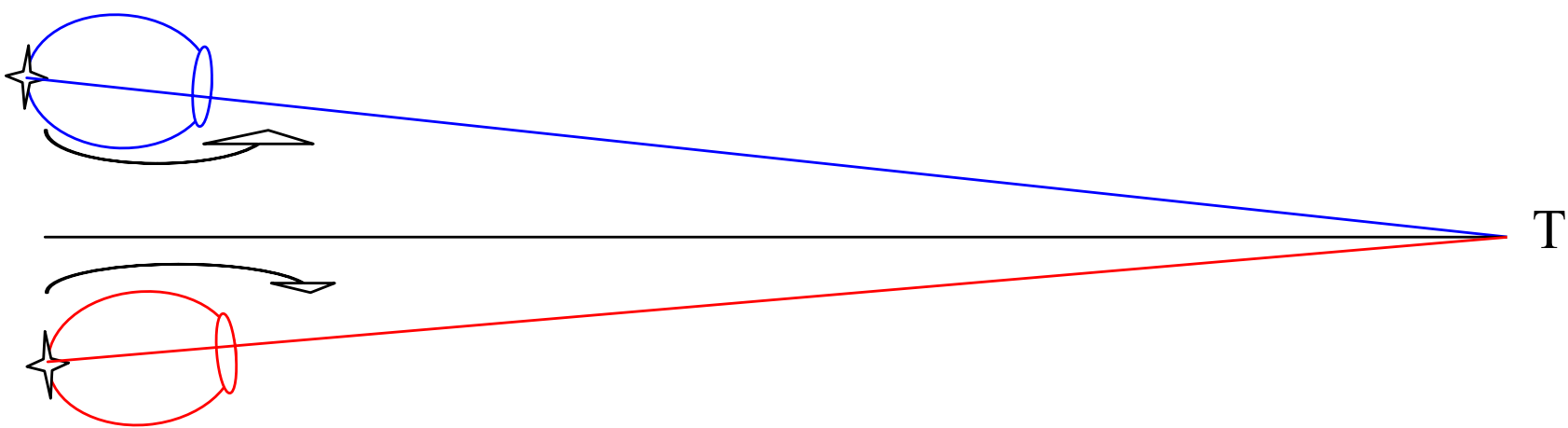

Figure 3. Compensatory vertical divergence (newly described eye movement). This eye movement (arrows) compensates for vertical transphoria by elevating the higher eye and depressing the lower eye by use of the opposing elevator and depressor muscles. This effectively brings the target image closer to the foveas. However, this creates increased stress and tension in the initial elevator and depressor muscles as well as the opposing compensatory elevator and depressor muscles. See Figure Key in Figure 1. 


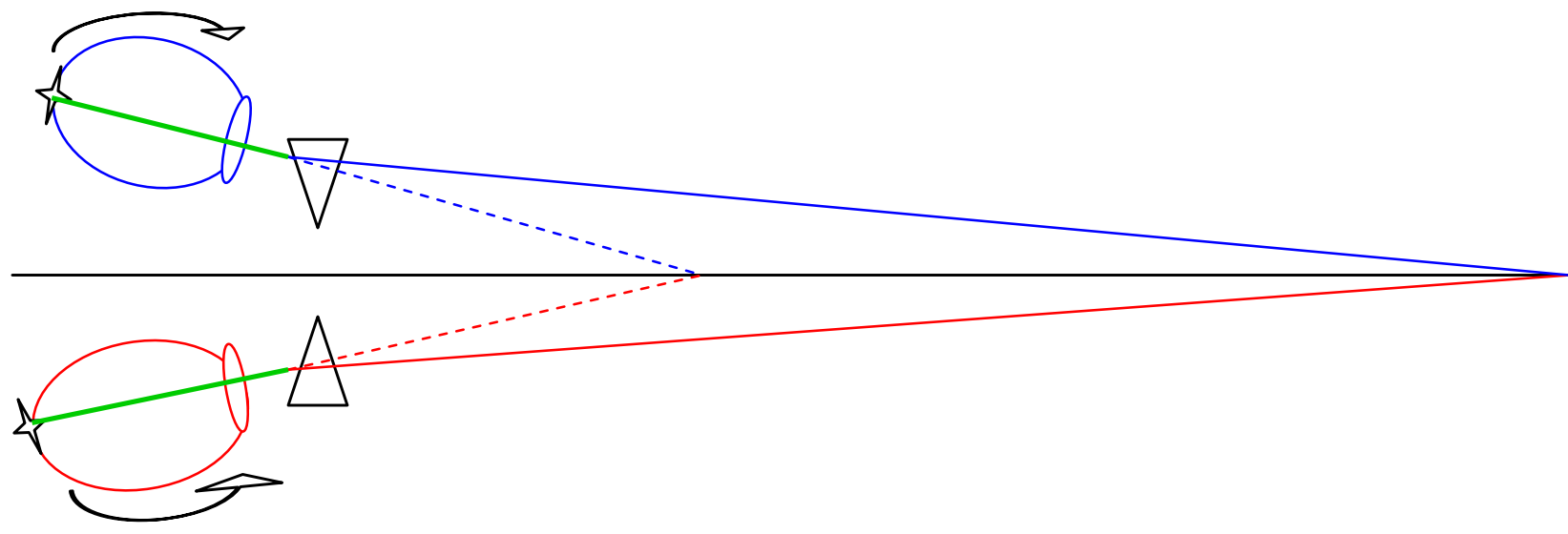

Figure 4. Prismatic correction of compensatory vertical divergence. Vertical prismatic lenses align the image from target with line of sight, eliminating the need for compensatory vertical divergence. This allows elimination of the use of the opposing compensatory elevator and depressor muscles, and the eyes to return to their transphoric position (arrows). This eliminates the extraocular muscle strain and fatigue, and concomitantly alleviates the symptoms of VH. See Figure Key in Figure 1.

and treating patients with $\mathrm{VH}$ as a result of using a diagnostic test that was more accurate in delineating the direction of vertical misalignment (prolonged monocular occlusion) as well as obtaining a more accurate vision prescription by the use of much-smaller units of prism in the spectacle lenses [13]. However, Roy's technique for diagnosis and treatment is not widely used, possibly because the procedure is time consuming for the practitioner and patient (each eye was occluded for 6 days) and there is little published evidence that small units of prism are valuable for treating vertical misalignment $[13,14]$.

\section{Diagnosis and Treatment of VH}

Historically, diagnosing and treating VH has been fraught with difficulty:

1. The range of symptoms is expansive and diverse, including pain (head and neck), vestibular (dizziness,

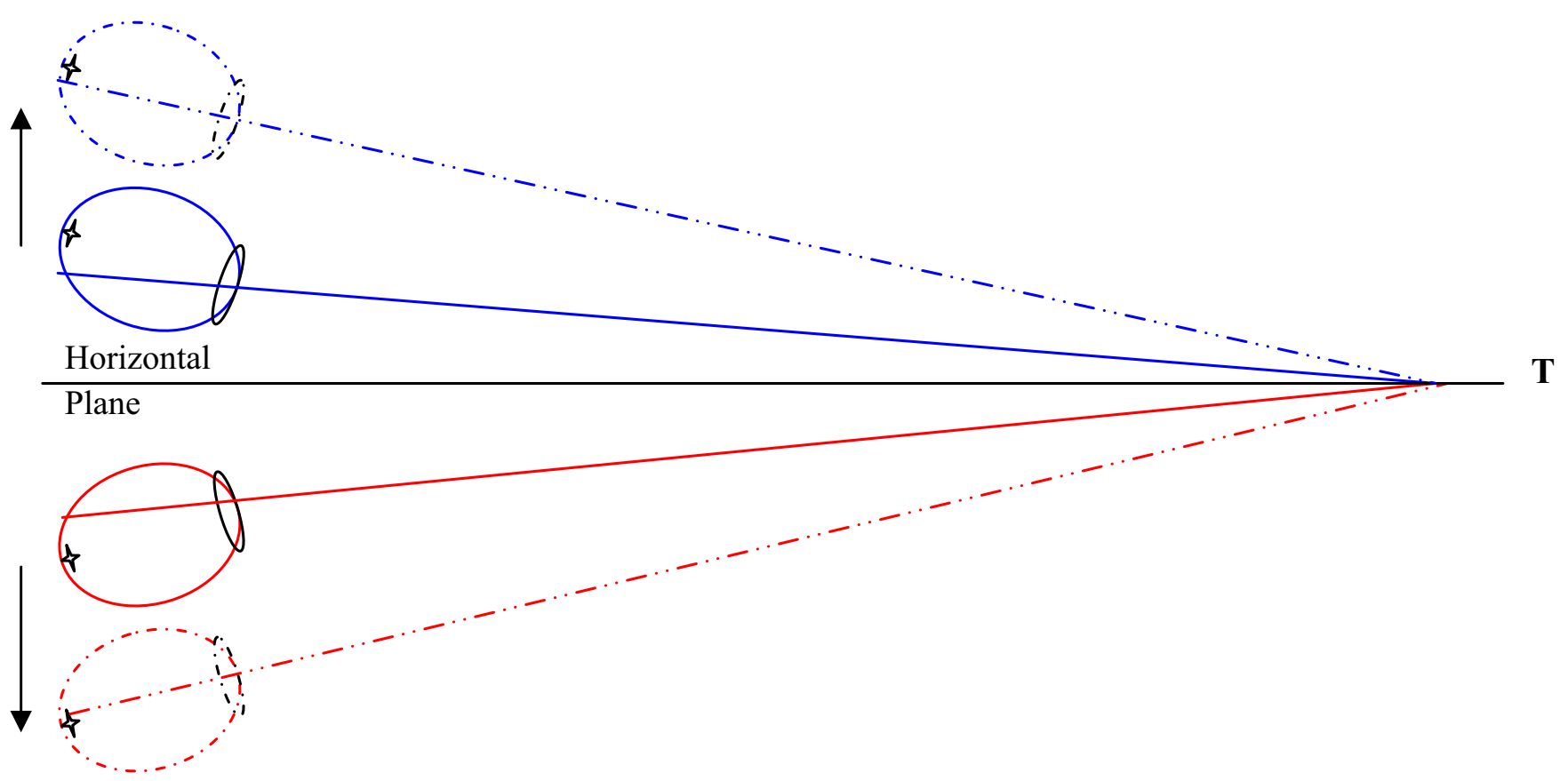

Figure 5. Effects of head tilt on the projection of an image onto the retina in $\mathrm{VH}$. Tilting the head increases the vertical separation of the eyes (ghosted images), which brings the target image closer to the foveas. See Figure Key in Figure 1. 
balance and gait, motion sickness), vision (blurred vision, reading difficulties, diplopia, light sensitivity), and psychiatric symptoms (anxiety, panic, agoraphobia) (Table 1) [2-11].

2. Although these symptoms individually are quite common, the symptom complex is not widely known by the general practitioner or the specialist to be associated with $\mathrm{VH}$, and these patients are frequently diagnosed with a host of other conditions (Table 1).

3. Tests designed to measure $\mathrm{VH}$ yield conflicting results that frequently do not correlate with the patient's symptoms [15-17].

4. The present consensus is that patients can accommodate to small amounts of $\mathrm{VH}$ without the need for assistance from prismatic corrective lenses, and therefore fractional units of prism are not believed to be needed or efficacious. Traditionally, large increments of prism (1-2 prism diopters) have been used to treat $\mathrm{VH}$.

Given the difficulties with testing and the manner in which vertical prism is currently being used (as delineated in the aforementioned points 3 and 4), it has been challenging to establish a relationship between the symptoms and the diagnosis of VH. Furthermore, these limitations have made the elucidation of the correct direction and amount of prism to prescribe difficult.

To overcome these obstacles, a 2-component process (on the basis of the history and physical examination) was developed to more accurately diagnose and treat $\mathrm{VH}$. The first component is a self-administered survey instrument (Table 4) designed to assess the severity of symptoms of patients suspected of having VH and to assess improvement of symptoms with treatment. Questions chosen for inclusion in the instrument represent all of the major symptom categories (head and eye pain, dizziness, reading difficulties, binocular vision symptoms, routine vision symptoms, and anxiety) because it has been noted that those patients experiencing VH have symptoms in many of the different categories simultaneously. The specific symptoms, the degree of symptom severity, and the degree of symptom frequency are different for each patient. In many cases, one symptom category is primary, whereas the other symptoms are adjunctive. This tool is presently undergoing validation studies.

The second component of the process is the determination of the direction and amount of vertical prism required. As previously mentioned, $\mathrm{VH}$ testing can be quite misleading. Our clinical experience indicates that a more accurate method to delineate the direction of the vertical misalignment is to assess the direction of the patients' head tilt. It is well known that patients with VH can manifest with a head tilt $[3,7]$. Our experience indicates that the eye that is physically higher as the result of the head tilt sees the higher image and requires base up prism for correction (Figures 2-4). This finding is in contrast to patients with a cranial nerve 4 (CN 4)
Table 4. VHS-Q: Initial and follow-up* version

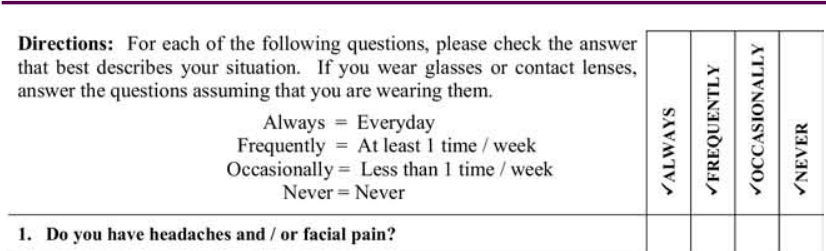

1. Do you have headaches and / or facial pain?

Draw in location of discomfort

(Scale 1-10: $1=$ extremely mild, $10=$ =xtremely severe)

2. Do you have pain in your eyes with eye movement?

3. Do you experience neck or shoulder discomfort?

4. Do you have dizziness and / or lightheadedness?

5. Do you experience dizziness, light-headedness, or nausea while performing close-up activities (i.e. - computer work, reading, writing)?

6. Do you experience dizziness, light-headedness, or nausea while performing far-distance activities (i.e. - driving, television, movies)?

7. Do you experience dizziness, light-headedness, or nausea when bending down and standing back up, or when getting up quickly from a seated position?

8. Do you feel unsteady with walking, or drift to one side while walking?

9. Do you feel overwhelmed or anxious while walking in a large department store (i.e. - Target, Wal-Mart, Meijer)?

10. Do you feel overwhelmed or anxious when in a crowd?

11. Does riding in a car make you feel dizzy or uncomfortable?

12. Do you experience anxiety or nervousness because of your dizziness?

Do you ever find yourself with your head tilted to one side?

14. Do you experience poor depth perception or have difficulty estimating distances accurately?

15. Do you experience double / overlapping / shadowed vision at far distances?

16. Do you experience double / overlapping / shadowed vision at near distances?

17. Do you experience glare or have sensitivity to bright lights?

18. Do you close or cover one eye with near or far tasks?

19. Do you skip lines or lose your place while reading (do you use your finger or a ruler or other guides to maintain your position on the page)?

20. Do you tire easily with close-up tasks (computer work, reading, writing)?

21. Do you experience blurred vision with far-distance activities (i.e. driving, television, movies, chalkboard at school)?

22. Do you experience blurred vision with close-up activities (i.e. - computer work, reading, writing)?

23. Do you blink to "clear up" distant objects after working at a desk or working with close-up activities (i.e. - computer work, reading, writing)?

24. Do you experience words running together with reading?

25. Do you experience difficulty with reading or reading comprehension? 2004-2010 Vision Specialists of Birmingham

*The Vertical Heterophoria Symptom Questionnaires (VHS-Q) - Follow-Up Version is identical to the Initial version except for the addition of one question:

"Compared to the way I felt before I came to Vision Specialists: If you are feeling better, by what percentage have you improved? \% improved"

How to score the VHS-Q Initial and Follow-Up version: The response given to the individual questions are given values of: Always $=3$, Frequently $=2$, Occasionally $=1$, and Never $=0$. A score is obtained by summing the values for all of the responses. Maximum score possible $=75$, minimum score $=0$. 


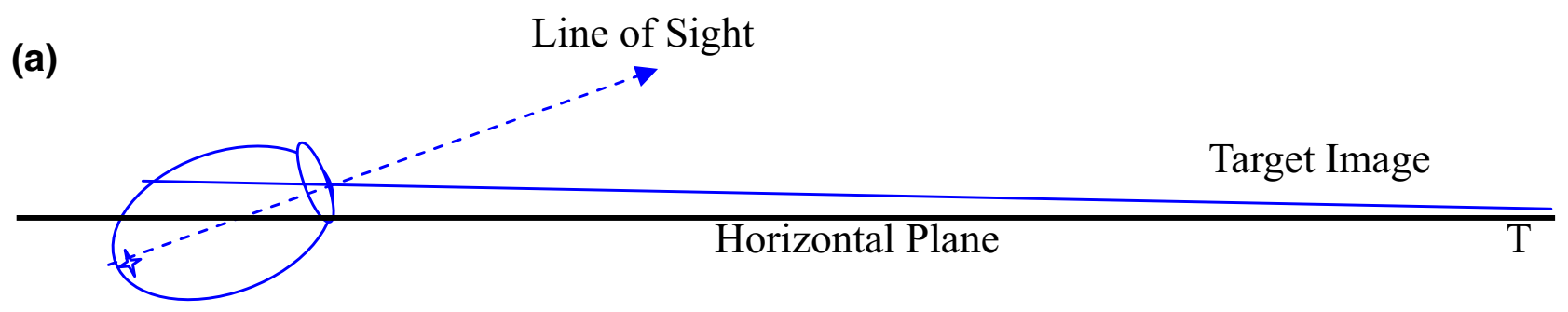

(b)

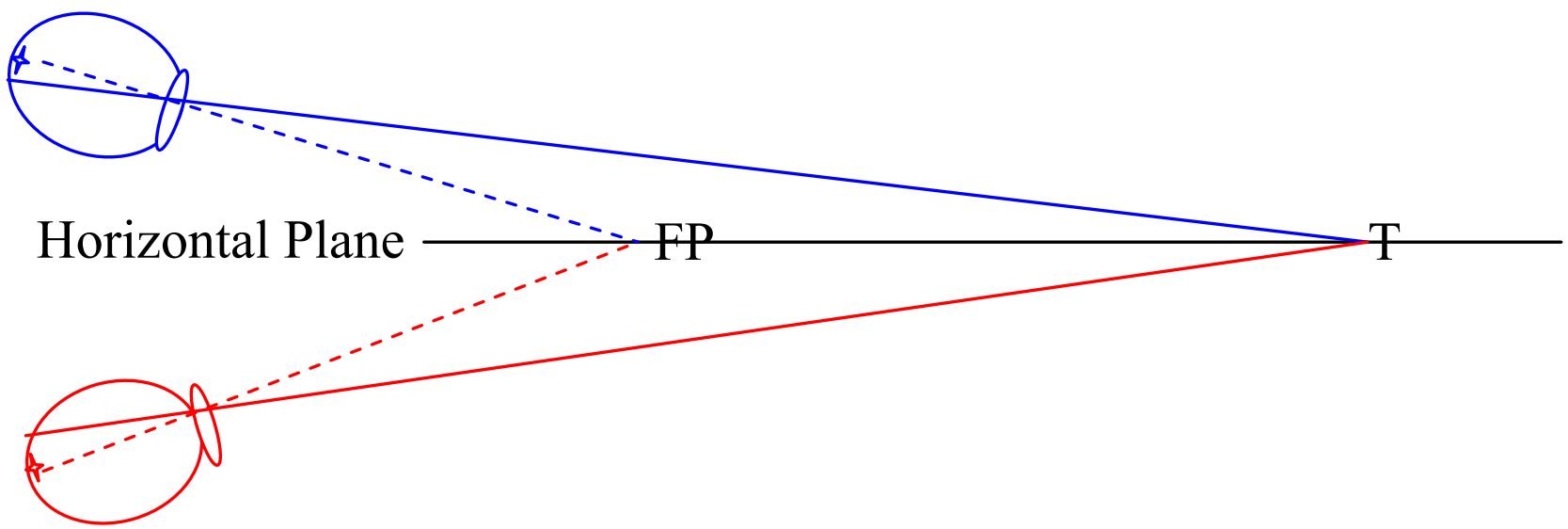

Figure 6. (A) Model of CN 4 injury/SO palsy. Pathology = elevation and external rotation of affected eye. The line of sight of the "physically high" eye is elevated. The target image strikes the retina above the fovea, which is interpreted by the brain as a low image. The head tilt elevates this eye even more to eliminate the torsional diplopia. (B) Model of VH. Pathology = depression of high eye, elevation of low eye (vertical transphoria). (Elucidated from the position of the head tilt, the position of the eye, the location of the image, and from the direction of correcting vertical prism.) The line of sight of the "physically high" eye is depressed. The target image strikes the retina below the fovea, which is interpreted by the brain as a high image. The head tilt elevates the high eye even more to eliminate the vertical disparity. See Figure Key in Figure 1.

injury or superior oblique (SO) muscle palsy, where the eye that is higher as the result of elevation from the injury or palsy sees the lower image and requires base down prism for correction (see Figure 6, 7).

Once the direction of the prismatic correction is elucidated, small units of vertical prism are incrementally added to the baseline prescription until $\mathrm{VH}$-associated symptoms are significantly reduced and comfort is maximized. This improvement is noted almost immediately and is maximized within 20 to 30 minutes of the patient wearing the appropriate lens prescription. Symptom reduction appears to remain stable over time (other VH patients have been followed by the author, who is an optometrist for as long as 10 years). Yearly examinations are required to assess for possible minor modifications, as occurs with any vision patient who is prescribed corrective lenses.

\section{Advantages of Prismatic Lenses in TBI Patients}

Prismatic lens treatment has several distinct advantages. The time required to optimize the initial individualized prismatic lens prescription and thereby maximize reduction of symptoms is brief (on average 2-3 months) and the average patient required 3 sets of lenses, making this diagnostic and therapeutic intervention cost effective and time efficient. Spectacle lenses and contact lenses are externally applied visual orthotics and are therefore noninvasive. With the reduction of symptoms, it was noted that some patients experienced significant progress in their other TBI-related therapy modalities, multiple medications frequently were discontinued, and fewer consultations and tests were ultimately required, leading to additional time and cost savings.

\section{Study Limitations}

There were several limitations to the present study. Many of the patients referred to the optometrist were unable to go for evaluation because of distance considerations (approximately 1 hour drive between offices) and insurance restrictions. Nearly half of the referred patients that were evaluated by the optometrist were excluded from analysis, which could have introduced severe bias. Because we are not studying a representative sample of all patients with TBI, we cannot 

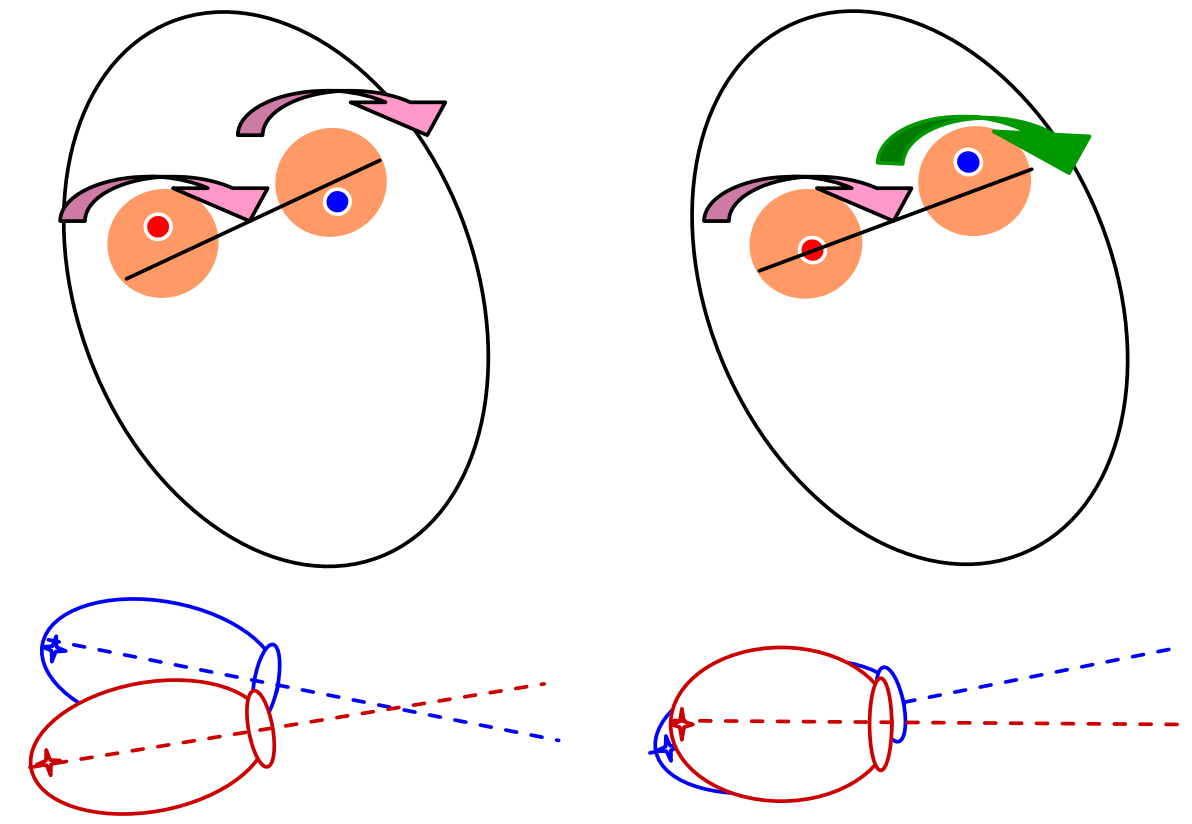

VH, orbital asymmetry model (which is optically equivalent to TBI model)

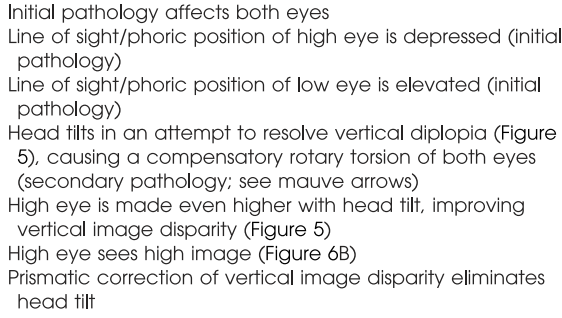

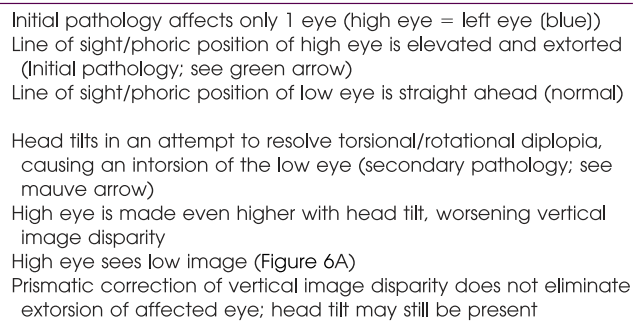

Figure 7. Comparison of pathophysiology of $\mathrm{VH}$ and $\mathrm{CN} 4$ Injury/SO palsy.

deduce the prevalence of VH in the TBI population (although anecdotally, it appeared to be quite prevalent). Because there was no placebo control and because the patients were not blinded to their treatment with prismatic lenses, it is possible that a placebo effect had an impact on the results. However, this is less likely in this group of patients because the effects of vertical prismatic lenses are not subtle. When the lenses are worn by those who require these lenses, noticeable relief of symptoms is obtained. Conversely, when these lenses are worn by those who do not require them, symptoms of VH develop, most notably nausea, anxiety/dysphoria, and dizziness. Another limitation was that there was only a single physiatrist and single optometrist involved in this study. Although this combination made for consistency, reproducibility was not established.

\section{CONCLUSION}

In this group of patients who developed postconcussive symptoms and VH symptoms secondary to their TBI, dys- function of the binocular visual system was found to be a single common factor shared by all patients. Treatment of the VH with individualized prismatic spectacle lenses was found to be effective in reducing symptom burden associated with both TBI and VH. These observations point to a relationship between TBI and binocular dysfunction and suggest that VH might be acquired from the TBI. Although this study was unable to establish the prevalence of $\mathrm{VH}$ in those with a TBI, anecdotally it appeared to be quite prevalent.

It is apparent that further research needs to be performed to further define the relationship between TBI and binocular dysfunction. Prospective randomized sham controlled trials to assess the effect of individualized prismatic spectacle lens treatment for TBI patients experiencing symptoms of VH would be beneficial. Studies designed to determine the effect prismatic lenses have on the amount of improvement a patient experiences with the standard TBI treatment modalities (like physical and occupational therapy), and whether prismatic lenses shorten the duration of these treatments would be important to perform. Establishing a relationship 
between TBI and $\mathrm{VH}$ and demonstrating effective treatment of post-TBI symptoms with individualized prismatic spectacle lenses would represent a new time efficient and costeffective approach in the evaluation and treatment of TBI patients.

\section{ACKNOWLEDGMENTS}

The authors thank Bonita Singal, MD, for assistance with statistical analysis and Bradford Walters, MD, for editorial assistance in manuscript preparation.

\section{REFERENCES}

1. Kraus J, Schaffer K, Ayers K, Stenehjem J, Shen H, Afifi AA. Physical complaints, medical service use, and social and employment changes following mild traumatic brain injury: A 6-month longitudinal study. J Head Trauma Rehabil 2005;20:239-256.

2. Borish IM. History and eye strain. In: Borish IM, ed. Clinical Refraction. 3rd ed. Chicago, IL: The Professional Press, Inc.; 1975, 327-338.

3. Borish IM. Analysis and prescription. In: Borish IM, ed. Clinical Refraction. 3rd ed. Chicago, IL: The Professional Press, Inc.; 1975, 866 ,

4. Bixenman WW. Vertical prisms. Why avoid them? Surv Ophthalmol 1984:29:70-78.

5. Schrier M. Practice notes on hyperphoria. BJOD 1997;5:68-69.
6. Roy RR. Symptomatology of binocular stress. Optom Wkly 1958;49: 907-912.

7. Duke-Elder S, Wybar K. Anomalies of binocular fixation. In: DukeElder S, Wybar K, eds. System of Ophthalmology. Vol. 6. Ocular Motility and Strabismus. St. Louis, MO: The C. V. Mosby Company; 1973, 536, 553.

8. Mitchell SW. Headaches and eye strain. Am J Med Sci 1876;71:363373.

9. Staab JP, Ruckenstein MJ. Expanding the differential diagnosis of chronic dizziness. Arch Otolaryngol Head Neck Surg 2007;133:170-176.

10. Furman JM, Jacob RG. Psychiatric dizziness. Neurology 1997;48:11611166.

11. Guerraz M, Yardley L, Bertholon $P$, et al. Visual vertigo: Symptom assessment, spatial orientation and postural control. Brain 2001;124: 1646-1656.

12. Stevens, GT. Functional nervous diseases. New York, NY: D. Appleton and Company; 1887, 200-203.

13. Roy RR. Ocular migraine and prolonged occlusion. Part 2. Optom Wkly 1953;44:1513-1518.

14. Wick BB. Prescribing vertical prism: How low can you go? J Optom Vision Dev 1997;28:77-85.

15. Gray LS. The prescribing of prisms in clinical practice. Graefes Arch Clin Exp Ophthalmol 2008;246:627-629

16. Schroeder TL, Rainey BB, Goss DA, Grosvenor TP. Reliability of and Comparisons among methods of measuring dissociated phorias. Optom Vis Sci 1996;73:389-397.

17. Gall R, Wick B. The symptomatic patient with normal phorias at distance and near: What tests detect a binocular vision problem? Optometry 2003;74:309-322.;74:309-322. 